\title{
Determinantes da implantação de um programa de segurança e saúde no trabalho
}

\author{
Sonia Cristina Lima Chaves, ${ }^{1}$ Vilma Sousa Santana, ${ }^{2}$ \\ Inez Cristina Martins de Leão, ${ }^{3}$ Jusiene Nogueira de Santana ${ }^{3}$ \\ e Lívia Maria Aragão de Almeida Lacerda ${ }^{3}$
}

Como citar Chaves SCL, Santana VS, Leão ICM, Santana JN, Almeida Lacerda LMA. Determinantes da implantação de um programa de segurança e saúde no trabalho. Rev Panam Salud Publica. 2009;25(3):204-12.

RESUMO Objetivo. Identificar preditores da implantação de um programa de saúde e segurança no trabalho (PSST) que integra a vigilância à saúde do trabalhador com a segurança no trabalho, envolvendo a participação de técnicos, empresários e trabalhadores.

Método. Neste estudo de desenho ecológico, foram estudadas empresas atendidas pelo PSST do Serviço Social da Indústria (SESI) no Estado da Bahia, selecionadas aleatoriamente, durante o ciclo entre 2005 e 2006. Os dados foram coletados em entrevistas com informantes chaves da empresa e relatórios técnicos do SESI. Com regressão linear múltipla, analisaram-se os fatores e subdimensões impulsionadores do PSST para a empresa, os trabalhadores e a equipe técnica do PSST.

Resultados. Das 78 empresas selecionadas (3 384 trabalhadores), 24,4\% haviam alcançado a implantação do PSST em grau avançado; 53,8\% em grau intermediário; e 19,3\% em grau incipiente. Fatores da empresa, dos trabalhadores e da equipe técnica se associaram positivamente ao grau de implantação do PSST $(\mathrm{P}<0,001)$. Destacou-se como o mais importante, isoladamente, a autonomia da gestão financeira do PSST $(\beta=4,40 ; \mathrm{P}<0,001)$. Na análise bivariada, associaram-se com a implantação o nível de conhecimento $(\beta=1,58 ; \mathrm{P}<0,05) e$ treinamento em SST dos trabalhadores $(\beta=0,40 ; \mathrm{P}<0,001)$ e a articulação entre a equipe de segurança $(\beta=1,89 ; \mathrm{P}<0,01)$ e a de saúde $(\beta=0,58 ; \mathrm{P}<0,05)$. Esses achados não se modificaram após ajuste por escolaridade dos trabalhadores e gestores, salários, grau de risco e porte das empresas.

Conclusões. A disponibilidade de recursos e de tempo dos trabalhadores para a saúde e a segurança no trabalho, a integração e o reforço de atividades de educação para trabalhadores e gestores e a melhor integração das equipes de saúde e segurança podem contribuir para o sucesso das ações de promoção da saúde nas empresas.

Palavras-chave Saúde ocupacional, segurança, gerência de segurança, vigilância, Brasil.

1 Faculdade de Odontologia, Departamento de Odontologia Social e Pediátrica. Correspondência: Avenida Araújo Pinho 62, $6^{\circ}$ andar, Canela, CEP 40110-912, Salvador, BA, Brasil. Fone: +55-71-3495. 6210; e-mail: schaves@ufba.br

2 Universidade Federal da Bahia (UFBa), Instituto de Saúde Coletiva, Programa Integrado em Saúde Ambiental e do Trabalhador (PISAT), Salvador (BA), Brasil.

3 Serviço Social da Indústria (SESI), Departamento Regional da Bahia, Salvador (BA), Brasil.
As doenças e os acidentes ocupacionais representam uma grande carga para os trabalhadores, as empresas, os serviços de saúde e a seguridade social. Os programas de intervenção para o enfrentamento desse problema se baseiam, em geral, em modelos de vigilância da saúde e da segurança, aplicados com diversas estratégias (1).
No Brasil, além da regulação e das normas e guias de boas práticas, são utilizados inspeções e penalidades, treinamentos e cursos que promovem o aprimoramento do desempenho das empresas na prática de proteção dos trabalhadores. O Ministério do Trabalho exige que todas as empresas com trabalhadores avaliem regularmente os riscos do 
ambiente de trabalho e a saúde de seus trabalhadores, sendo que os resultados dessas avaliações devem subsidiar os programas de prevenção. Os dois programas obrigatórios para empresas são o Programa de Prevenção de Riscos Ambientais (PPRA) (Norma Regulamentadora No. 9, ou NR-9) (2) e o Programa de Controle Médico e Saúde Ocupacional (PCMSO) (NR-7) (3).

As intervenções baseadas em diagnósticos situacionais são positivas porque geram decisões fundamentadas em informação e valorizam a participação dos trabalhadores. Todavia, apresentam falhas na própria concepção, como a separação entre agentes de risco e efeitos sobre a saúde, o que resulta em desarticulação das ações e em tensões e vieses disciplinares em um campo essencialmente interdisciplinar.

Poucos estudos têm se debruçado sobre a avaliação de programas de saúde dos trabalhadores em empresas. Em um desses trabalhos (4), os principais determinantes de clima de segurança percebidos pelos trabalhadores foram as condições ambientais, a existência de programas de segurança, o apoio organizacional e a comunicação na empresa. Em países orientais, certas avaliações de programas de saúde e segurança realizadas em pequenas empresas revelaram que a associação de empresas por ramo de atividade reforça o compromisso com a segurança. Além disso, foi observado que o uso de estratégias baseadas em metas e resultados, por parte das empresas, resulta em um uso efetivo de checklists, na realização de trabalhos de grupo (5) e na aplicação de boas práticas adaptadas a condições locais, como, por exemplo, atividades de baixo custo (6).

Uma pesquisa sobre fatores preditores de acidentes de trabalho identificou que, em nível individual, os mais importantes foram escolaridade, experiência, aprendizado e atitudes dos trabalhadores (7). Em um estudo conduzido nos Estados Unidos, verificou-se que a maioria das empresas não avaliava a efetividade de seus programas de vigilância. Entretanto, o estudo revelou que esses programas tinham efeitos positivos, inclusive a redução de custos com saúde, o aumento da detecção precoce de problemas de saúde, a diminuição da ocorrência de agravos e enfermidades e o aumento da produtividade (8).

Além de raros, os estudos que avaliam os programas de saúde ocupacional en- focam desfechos ou programas específicos de intervenção sobre agentes de risco ou enfermidades e agravos isolados e, em geral, apresentam problemas metodológicos (9). No Brasil, a avaliação de programas de saúde ocupacional é incipiente. Miranda e Dias (10) estudaram PPRA e PCMSO de 30 empresas e verificaram que os relatórios de risco ambiental $(92,9 \%)$ e os de problemas de saúde dos trabalhadores $(85,7 \%)$ apresentavam inconsistências em relação ao grau de risco das empresas envolvidas; também foi constatada a baixa qualidade técnica desses relatórios. Esses autores constataram ainda que nenhuma empresa havia envolvido os trabalhadores na elaboração dos programas. Em uma avaliação sobre o controle do ruído em PPRA/ PCMSO de indústrias de São Paulo, além da inexistência de mensurações, observou-se que não eram emitidas comunicações de acidentes de trabalho (CAT) (11), que são registros para fins de seguridade social no Ministério da Previdência Social. Com base em dados semelhantes, outro estudo apontou que as avaliações clínicas do PCMSO não abrangiam enfermidades ou agravos ocupacionais e que era limitado o registro de agentes de risco ocupacional (12). Portanto, as evidências apontam para a insuficiência da vigilância à saúde ocupacional nas empresas e para a falta de cumprimento das recomendações legais.

A avaliação de programas de saúde comumente abrange os componentes de implantação, estrutura, processo e desfechos ou resultados (13). Embora a avaliação da implantação, ou seja, do grau pelo qual a proposta planejada foi efetivada, seja fundamental para determinar o cumprimento dos impactos esperados, são raros os estudos com esse foco. Entretanto, o conhecimento dos determinantes de implantação de programas de saúde, ou seja, dos fatores que influenciam a operacionalização adequada de uma intervenção (14), pode contribuir para a identificação de obstáculos ou fatores propulsores que podem potencialmente ser gerenciados visando ao alcance das metas desejadas. No único estudo encontrado sobre avaliação de implantação de programas de saúde e segurança, realizado na Noruega, observou-se que o grau de implantação aumentou no período de estudo e foi menor nas empresas de pequeno porte e privadas. Os principais preditores do grau de implantação foram o treinamento dos trabalhadores e dos gestores em segurança, a elaboração de planos de ação e a realização de inspeções em locais de trabalho (15).

No Estado da Bahia, o Serviço Nacional da Indústria (SESI) desenvolve um PSST que em 2006 atingiu 194 empresas, estando prevista a sua avaliação periódica. Esse programa tem sua origem em 1994, quando, após a divulgação das NR-7 e NR-9, o SESI Departamento Regional da Bahia avaliou suas práticas em saúde e segurança e identificou problemas como a falta de planejamento, o atendimento fragmentado à demanda espontânea sem consideração às necessidades dos trabalhadores, a grande descontinuidade das ações e um enfoque predominantemente clínico, sem atenção à prevenção ou promoção da saúde. Elaborou-se, então, um programa de saúde ocupacional baseado na vigilância à saúde, com eixo na integração das ações de saúde, higiene e segurança, participação de equipes multiprofissionais, envolvimento da empresa e trabalhadores e proposição de intervenções adequadas às necessidades. Esse programa sofreu redefinições sucessivas, alcançando o formato atual a partir de 2004.

O objetivo deste estudo foi identificar os fatores que se associaram positivamente ao sucesso na implantação do PSST proposto pelo SESI em empresas do Estado da Bahia.

\section{MATERIAIS E MÉTODOS}

Este é um estudo de desenho ecológico conduzido com dados agregados por empresa, relativos ao registro da implantação do PSST no ciclo entre 2005 e 2006, segundo a avaliação do grau de implantação proposta por Denis e Champagne (14). O PSST analisado atende 11471 trabalhadores de indústrias manufatureiras e 3822 de empresas da construção civil no Estado da Bahia, segundo dados da Relação Anual de Informações Sociais (RAIS) para o ano de 2004.

A avaliação do PSST parte de um modelo lógico de intervenção $(16,17)$, composto de três níveis: a) contexto de implantação; b) implantação da intervenção; e c) resultados. Compreende-se como implantação um processo de gestão do início do programa que envolve várias etapas, cujo final é o programa implantado com todas as atividades previstas em pleno funcionamento. A implantação do PSST se inicia com a etapa de sensibilização do empresário ou 
pessoa focal da empresa, indicada, em geral, pelo dirigente principal. Após a sensibilização, realizada por meio de visitas e reuniões com representantes do SESI-Bahia, instituição prestadora do serviço, apresentam-se o propósito e a estratégia do PSST e as vantagens relativas à adesão aos marcos regulatórios do Ministério do Trabalho e Emprego, à política de saúde do Ministério da Saúde e aos subsídios oferecidos pelo SESI relativos a esse programa. Com a aceitação, iniciam-se as atividades, com uma visita inicial para levantamento de dados qualitativos, seguida de procedimentos para realização de avaliações ambientais com vistas à identificação dos grupos homogêneos de exposição. Propõe-se um plano de ação conjunto da equipe. As visitas dos profissionais de saúde e segurança são realizadas periodicamente para acompanhamento das sugestões propostas e realização de atividades no local de trabalho. Os trabalhadores realizam exames médicos que compõem um relatório epidemiológico da empresa. Para uma implantação bem-sucedida, considerou-se que haveria fatores impulsionadores no âmbito da empresa, entre os trabalhadores e na equipe do SESI responsável pelo PSST.

Outros fatores não diretamente integrantes do programa, como o nível de escolaridade médio das pessoas focais e dos trabalhadores, os salários, o grau de risco ocupacional da empresa de acordo com a NR-9 e o porte da empresa, também poderiam introduzir modificações nos vetores impulsionadores da implantação. Como evidências da situação final de implantação do PSST consideraramse a efetivação do diagnóstico epidemiológico e de riscos ambientais, a execução adequada do PCMSO e PPRA, a realização de atividades de vigilância à saúde com a incorporação dos resultados do diagnóstico na programação e a divulgação das informações para gestores e trabalhadores, no ciclo de tempo de implantação de 1 ano. Além disso, foi ainda considerado o uso adequado dessas informações pela empresa na consolidação das ações de segurança e saúde no âmbito local. Do modelo lógico, derivou-se uma matriz de dimensões e subdimensões com seus respectivos componentes. Cada um desses níveis compreende dimensões definidas a partir do modelo lógico e dos padrões operacionais propostos nos documentos do programa (tabela 1).
De uma lista de todas as empresas participantes, selecionaram-se, de modo aleatório, por alocação proporcional dos sub-ramos mais comuns de atividade econômica, 80 empresas (3 384 trabalhadores) (18). Como parte das obrigações contratuais, cada empresa indicava uma pessoa focal para contatos com a prestadora. Em geral, a pessoa focal era o gerente operacional. De acordo com a programação das atividades, tendo a empresa completado um ciclo de implantação, dava-se início à avaliação da etapa correspondente. Para tal, eram colhidas informações com a pessoa focal sobre os fatores impulsionadores da empresa e com membros da equipe de saúde e segurança da empresa sobre fatores impulsionadores dos trabalhadores e da prestadora. Portanto, foi necessária a elaboração de formulários para cada um dos tipos de respondente, pessoa focal e equipe SST. Os formulários destinados à pessoa focal foram preenchidos por meio de entrevistas telefônicas, fax ou visitas individuais. As equipes de SST respondiam às questões presencialmente, com narrativas escritas.

Os instrumentos empregados continham perguntas relativas a cada dimensão, subdimensão e a seus respectivos componentes específicos, de acordo com o modelo lógico do programa. Foram consideradas quatro dimensões (tabela 1): fatores impulsionadores para SST na empresa; fatores impulsionadores à SST entre os trabalhadores; fatores impulsionadores da prestadora do serviço de SST, no caso o SESI; e grau de implantação do PSST. Os formulários preenchidos foram lidos e analisados pela equipe da avaliação, coletivamente, em oficinas de trabalho específicas para atribuição dos escores para cada subdimensão e respectivos critérios. Os critérios empregados para a atribuição dos escores eram qualitativos para componentes subjetivos e quantitativos quando se utilizavam medidas de cumprimento de metas, por exemplo. Esses escores eram discutidos até se alcançar um consenso sobre a pontuação a ser atribuída àquela dimensão.

A implantação, por sua vez, foi classificada nas seguintes categorias: implantação avançada: 75 a 100\% do escore máximo proposto; implantação intermediária: 51 a $75 \%$ do escore máximo proposto; implantação incipiente: 26 a 50\% do escore máximo; não implantado: menos de $25 \%$ do escore máximo proposto para a dimensão grau de implantação do PSST (tabela 1).
A variável dependente foi a dimensão grau de implantação do PSST. Os escores correspondentes das subdimensões foram somados, resultando em um escore global do grau de implantação do PSST, analisado como variável contínua. Esse escore global varia, portanto, entre 0 e 100, considerando-se o PSST completamente implantado quando atingisse o valor máximo.

As variáveis preditoras foram as três dimensões restantes, ou seja, fatores impulsionadores da empresa, fatores impulsionadores entre os trabalhadores e fatores impulsionadores da prestadora de serviços. Também nesse caso o escore global para cada dimensão foi calculado pelo somatório dos escores atribuídos a cada uma das subdimensões; os escores das subdimensões, por sua vez, representam a soma dos escores atribuídos a cada critério (tabela 1).

As covariáveis foram também definidas a partir de escores atribuídos pela equipe com base nas evidências da implantação do programa na empresa. Foram consideradas como covariáveis potencialmente modificadoras do efeito, ou confundidoras, as seguintes: 1) escolaridade da pessoa focal, que variou de ensino fundamental (escore zero) até nível superior (escore 5); 2) a escolaridade dos trabalhadores, que variou de $0 \%$ de trabalhadores com ensino secundário completo ou mais (escore zero) a $100 \%$ com ensino secundário completo ou mais (escore 15); 3) o salário dos trabalhadores, de $10 \%$ dos trabalhadores com saláriobase superior ao salário mínimo (escore 0) a $100 \%$ com salário-base superior ao mínimo (escore 10). A covariável grau de risco seguiu a classificação definida pelo Ministério do Trabalho na NR-9, de I a IV. O porte da empresa foi classificado com base no número absoluto de empregados: < 20; entre 20 e 50; > 50 .

\section{Análise estatística}

A associação entre os fatores impulsionadores e o grau de implantação do PSST foi estimada com coeficientes dos modelos de regressão linear múltipla, verificando-se o nível de significância estatística dos coeficientes de regressão para as dimensões, subdimensões e componentes (critérios) e os $\mathrm{R}$ quadrados. A modelagem foi realizada com cada variável preditora separadamente e em seguida conjuntamente, por componente, para verificar o efeito isolado, indepen- 
TABELA 1. Fatores preditores do grau de implantação do Programa de Saúde e Segurança no Trabalho do Serviço Social da Indústria no Estado da Bahia, Brasil, 2007

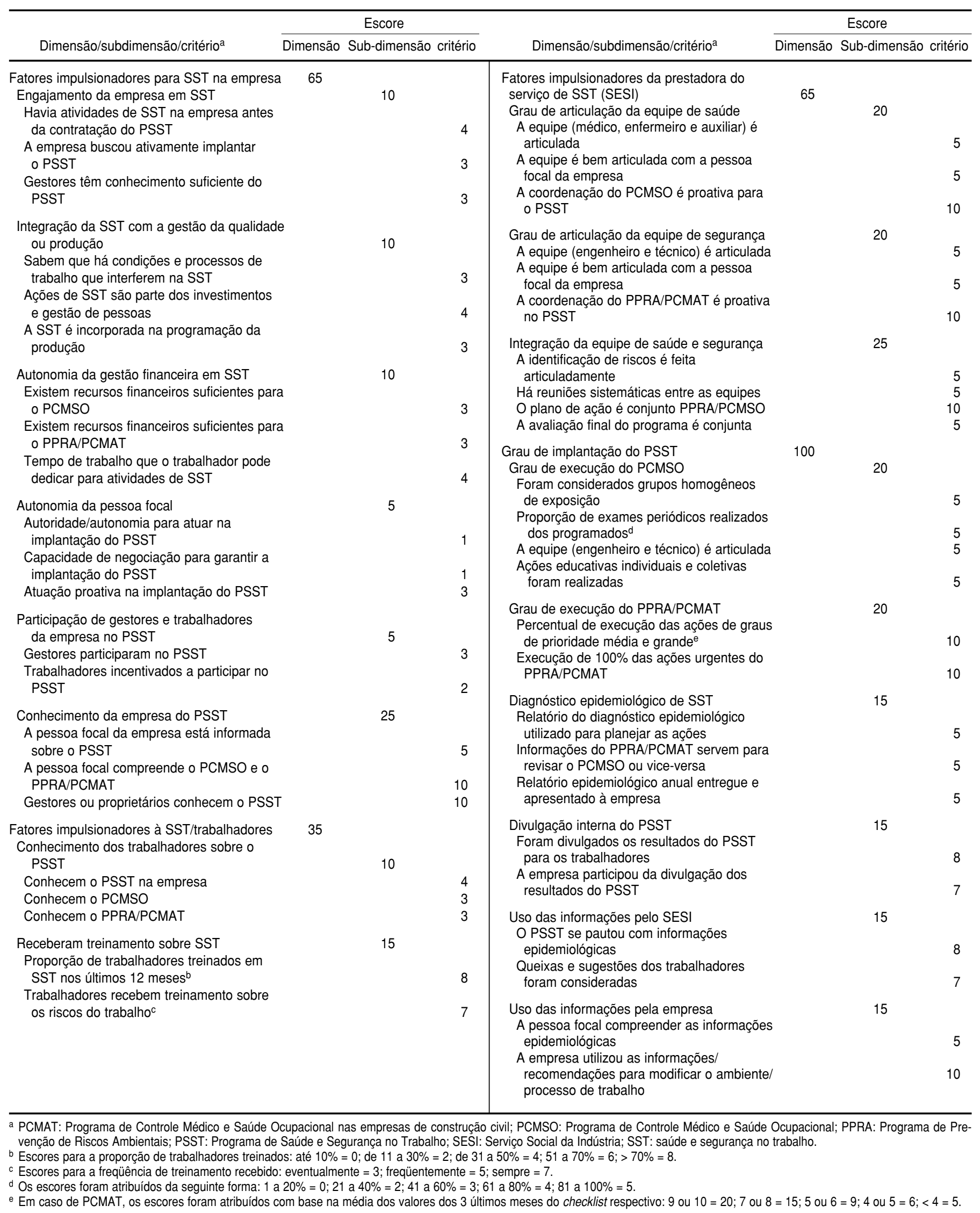


dente dos demais. As covariáveis foram testadas para confundimento, isto é, verificando-se diferenças de pelo menos $20 \%$ entre os coeficientes de regressão dos modelos saturados e sem a covariável em análise. Termos-produto e testes de razão de verossimilhança foram empregados para identificar modificadores de efeito, para um alfa $=0,05$. Na modelagem, as covariáveis foram tratadas em sua forma escalar, utilizando-se os escores respectivos, e nas tabelas de freqüência, em seu formato dicotômico. A base de dados foi criada com o Epi Info versão 6.0. A análise foi realizada com o SAS versão 9.0. Realizou-se a análise de resíduos dos modelos e todas as covariáveis foram tratadas em sua forma original contínua, exceto o grau de risco NR-9, para o qual consideraram-se as respectivas variáveis de desenho.

O estudo foi conduzido com informações de rotina dos serviços de saúde ocupacional do SESI, que autorizou o uso dos dados e a sua identificação nas publicações. Como se tratam de dados administrativos, não individuais e anônimos, não se submeteu o protocolo para apreciação por um Comitê de Ética em Pesquisa.

\section{RESULTADOS}

Das 80 empresas selecionadas, uma foi excluída por falência e outra por término do contrato com o SESI, totalizando duas perdas $(2,5 \%)$. Das 78 empresas que compuseram a população do estudo, verificou-se que $42(53,8 \%)$ foram classificadas no grau intermediário de implantação, 19 no grau avançado $(24,4 \%)$ e $15(19,2 \%)$ consideradas como tendo implantação incipiente. Em duas empresas $(2,6 \%)$, o PSST foi considerado não implantado.

Na tabela 2 apresentam-se as características das empresas de acordo com o grau de risco ocupacional NR-9. As em- presas não diferiram grandemente em relação à escolaridade da pessoa focal. Todavia, as empresas de risco IV apresentaram o maior número de trabalhadores com escolaridade maior, enquanto que as de risco III tinham salários mais altos. A tabela 2 mostra ainda que, dentre os fatores impulsionadores examinados, as empresas de menor risco (I e II) tiveram maior proporção de fatores favoráveis à implantação de parte da própria empresa e reduzida contribuição dos trabalhadores em comparação às empresas nos demais grupos de risco. Houve uma tendência a um desenvolvimento mais elevado da implantação entre as de grau de risco IV. As empresas de menor risco apresentaram pessoa focal e trabalhadores com menor escolaridade, salários mais baixos e número menor de trabalhadores.

A análise de regressão linear múltipla mostrou que todos os fatores impulsionadores - da empresa, dos trabalhadores

TABELA 2. Características das empresas de acordo com o grau de risco, estudo sobre o grau de implantação do Programa de e Saúde e Segurança no Trabalho do Serviço Social da Indústria no Estado da Bahia, Brasil, 2007

\begin{tabular}{|c|c|c|c|c|c|c|c|c|}
\hline \multirow[b]{3}{*}{ Variável } & \multicolumn{6}{|c|}{ Grau de risco } & & \\
\hline & \multicolumn{2}{|c|}{$\begin{array}{c}\text { I e II } \\
(n=14)\end{array}$} & \multicolumn{2}{|c|}{$\begin{array}{c}\text { III } \\
(n=44)\end{array}$} & \multicolumn{2}{|c|}{$\begin{array}{c}\text { IV } \\
(n=20)\end{array}$} & \multicolumn{2}{|c|}{$\begin{array}{c}\text { Total } \\
(n=78)\end{array}$} \\
\hline & No. & $\%$ & No. & $\%$ & No. & $\%$ & No. & $\%$ \\
\hline \multicolumn{9}{|c|}{ Escolaridade/pessoa focal ${ }^{a}$} \\
\hline Baixa (< secundário) & 9 & 64,3 & 23 & 52,3 & 11 & 55,0 & 43 & 55,1 \\
\hline Alta (superior) & 5 & 35,7 & 21 & 47,7 & 9 & 45,0 & 35 & 44,9 \\
\hline \multicolumn{9}{|c|}{ Escolaridade do trabalhador ${ }^{a}$} \\
\hline Baixa & 11 & 78,6 & 32 & 72,7 & 10 & 50,0 & 63 & 68,0 \\
\hline Alta & 3 & 21,4 & 12 & 27,3 & 10 & 50,0 & 25 & 32,0 \\
\hline \multicolumn{9}{|l|}{ Salário do trabalhador ${ }^{\mathrm{a}}$} \\
\hline Baixo & 10 & 71,4 & 25 & 56,8 & 14 & 70,0 & 49 & 62,8 \\
\hline Alto & 4 & 28,6 & 19 & 43,2 & 6 & 30,0 & 29 & 37,2 \\
\hline \multicolumn{9}{|l|}{ No. de trabalhadores } \\
\hline$<20$ & 5 & 35,7 & 19 & 43,2 & 6 & 30,0 & 30 & 38,5 \\
\hline 20 a 50 & & 57,2 & 13 & 29.6 & 7 & 35,0 & 28 & 35,9 \\
\hline$>50$ & 1 & 7,1 & 12 & 27,3 & 7 & 35,0 & 20 & 25,6 \\
\hline \multicolumn{9}{|c|}{ Impulsionadores empresa ${ }^{b}$} \\
\hline Baixo & 5 & 35,7 & 16 & 36,4 & 5 & 25,0 & 26 & 33,3 \\
\hline Médio & 2 & 14,3 & 14 & 31,8 & 10 & 50,0 & 26 & 33,3 \\
\hline Alto & 7 & 50,0 & 14 & 31,8 & 5 & 25,0 & 26 & 33,3 \\
\hline \multicolumn{9}{|c|}{ Impulsionadores trabalhadores ${ }^{b}$} \\
\hline Baixo & 5 & 35,7 & 20 & 45,5 & 1 & 5,0 & 26 & 33,3 \\
\hline Médio & 6 & 42,9 & 11 & 25,0 & 9 & 45,0 & 26 & 33,3 \\
\hline Alto & 3 & 21,4 & 13 & 29,6 & 10 & 50,0 & 26 & 33,3 \\
\hline \multicolumn{9}{|c|}{ Impulsionadores equipes SST ${ }^{b, c}$} \\
\hline Baixo & 1 & 7,1 & 20 & 45,5 & 6 & 30,0 & 26 & 33,3 \\
\hline Médio & & 64,3 & 10 & 22,7 & 8 & 40,0 & 26 & 33,3 \\
\hline Alto & 4 & 28,6 & 14 & 31,8 & 6 & 30,0 & 26 & 33,3 \\
\hline \multicolumn{9}{|c|}{ Grau de implantação do PSST ${ }^{\text {b,d }}$} \\
\hline Incipiente & 4 & 28,6 & 18 & 40,9 & 5 & 25,0 & 27 & 34,6 \\
\hline Intermediário & 4 & 28,6 & 16 & 36,4 & 5 & 25,0 & 25 & 32,0 \\
\hline Avançado & 6 & 42,9 & 10 & 22,7 & 10 & 50,0 & 26 & 33,3 \\
\hline
\end{tabular}


e da equipe prestadora - foram positivamente associados com o grau de implantação $(P<0,001)$ quando analisados separadamente (tabela 3). Quando os componentes (conjunto de critérios) de cada subdimensão foram considerados no modelo, verificou-se que, entre os fatores da empresa, apenas a autonomia da gestão financeira permaneceu estatisticamente significativa $(\beta=4,40 ; P<0,001)$. $\mathrm{Na}$ análise conjunta dos fatores relativos ao trabalhador, o conhecimento $(\beta=1,58$; $P<0,05)$ e o treinamento $(\beta=0,40$; $P<0,001)$, foram ambas preditores independentes do grau da implantação, enquanto que, dentre os fatores da equipe de SST da prestadora, a articulação da equipe de segurança $(\beta=1,89 ; P<0,01)$ e a integração $(\beta=0,58 ; P<0,05)$ desta com a de saúde foram os principais fatores para o sucesso da implantação. Não houve evidências de interação estatística entre as subdimensões em análise (tabela 3).

Na tabela 4 os resultados da modelagem revelam que, quando analisados simultaneamente, apenas os fatores da
TABELA 3. Regressão linear para as subdimensões, estudo sobre grau de implantação do Programa de Saúde e Segurança no Trabalho do Serviço Social da Indústria no Estado da Bahia, Brasil, 2007

\begin{tabular}{lcc}
\hline & \multicolumn{2}{c}{ Análise univariada } \\
\cline { 2 - 3 } Modelos/subdimensões e componentes & $\beta_{\text {bruto }}$ & $\beta_{\text {ajustado }}$ \\
\hline Fatores da empresa (escore global) & $1,02^{\mathrm{c}}$ & - \\
Engajamento da empresa em SST & $2,22^{\mathrm{d}}$ & 0,14 \\
Integração da SST com a produção & $3,15^{\mathrm{c}}$ & 0,11 \\
Autonomia da gestão financeira & $5,52^{\mathrm{c}}$ & $4,40^{\mathrm{b}}$ \\
Autonomia da pessoa focal & $4,77^{\mathrm{c}}$ & 1,19 \\
Participação de gestores e trabalhadores no PSST & $5,03^{\mathrm{c}}$ & 1,32 \\
Conhecimento dos gestores sobre SST & $1,41^{\mathrm{c}}$ & 0,34 \\
& & - \\
Fatores do trabalhador (escore global) & $1,57^{\mathrm{c}}$ & $1,58^{\mathrm{d}}$ \\
Conhecimento sobre PSST & $2,54^{\mathrm{c}}$ & $0,40^{\mathrm{b}}$ \\
Treinamento em SST & $1,87^{\mathrm{c}}$ & - \\
& & 0,71 \\
Fatores da equipe de SST prestadora (escore global) & $0,74^{\mathrm{c}}$ & $1,89^{\mathrm{e}}$ \\
Grau de articulação da equipe saúde & 0,73 & $0,58^{\mathrm{d}}$ \\
Grau de articulação da equipe segurança & $2,08^{\mathrm{c}}$ \\
Integração da equipe & $0,70^{\mathrm{d}}$ & \\
\hline
\end{tabular}

a SST: saúde e segurança no trabalho. PSST: Programa de Saúde e Segurança no Trabalho.

${ }^{\mathrm{b}} \beta_{\text {aiustado }}=$ coeficiente de regressão da equação contendo todos os componentes de cada subdimensão, separadamente. 0 coeficiente é ajustado para as variáveis que integram cada subdimensão.

c $P<0,001$.

d $P=0,05$.

e $P=0,01$.

TABELA 4. Coeficientes da regressão linear $\left(\beta_{\mathrm{i}}\right)$ para escores globais das subdimensões ajustados pelas covariáveis de contexto, estudo sobre grau de implantação do Programa de Saúde Segurança no Trabalho do Serviço Social da Indústria no Estado da Bahia, Brasil, 2007

\begin{tabular}{|c|c|c|}
\hline Modelos (escore globais das subdimensões analisadas) ${ }^{a}$ & $\beta_{\mathrm{i}}$ & $\mathrm{R}^{2}$ ajustado \\
\hline $\begin{array}{l}\text { Modelo } 1 \\
\text { Fatores da empresa } \\
\text { Fatores do trabalhador } \\
\text { Fatores da equipe de SST prestadora }\end{array}$ & $\begin{array}{r}0,71^{b} \\
0,83^{c} \\
0,12 \\
F=18,85\end{array}$ & $\begin{array}{r}0,4102 \\
P<0,0001\end{array}$ \\
\hline $\begin{array}{l}\text { Modelo } 2 \text { (ajustado por escolaridade da pessoa focal e do trabalhador) } \\
\text { Fatores da empresa } \\
\text { Fatores do trabalhador } \\
\text { Fatores da equipe de SST prestadora }\end{array}$ & $\begin{array}{r}0,70^{b} \\
0,90^{c} \\
0,14 \\
F=11,24\end{array}$ & $\begin{array}{r}0,3995 \\
P<0,0001\end{array}$ \\
\hline $\begin{array}{l}\text { Modelo } 3 \text { (ajustado escolaridade da pessoa focal e do trabalhador e por salário) } \\
\text { Fatores da empresa } \\
\text { Fatores do trabalhador } \\
\text { Fatores da equipe de SST prestadora }\end{array}$ & $\mathrm{F}=\mathrm{O}=\begin{aligned} & 0,70^{\mathrm{b}} \\
& 0,91^{\mathrm{c}} \\
& 9,13\end{aligned}$ & $P<0,0001$ \\
\hline $\begin{array}{l}\text { Modelo } 4 \text { (ajustado por nível de escolaridade da pessoa focal e do trabalhador, } \\
\text { salário e grupo de risco da empresa) } \\
\text { Fatores da empresa } \\
\text { Fatores do trabalhador } \\
\text { Fatores da equipe de SST prestadora }\end{array}$ & $\begin{aligned} & 0,72^{b} \\
& 0,86^{d} \\
& 0,16 \\
= & 7,95\end{aligned}$ & $P<0,0001$ \\
\hline $\begin{array}{l}\text { Modelo } 5 \text { (ajustado por nível de escolaridade da pessoa focal e do trabalhador, } \\
\text { salário, grupo de risco e porte da empresa) } \\
\text { Fatores da empresa } \\
\text { Fatores do trabalhador } \\
\text { Fatores da equipe de SST prestadora }\end{array}$ & $\begin{aligned} & 0,70^{b} \\
& 0,85^{c} \\
& 0,23 \\
& F= 6,23\end{aligned}$ & $\begin{array}{r}0,3675 \\
P<0,0001\end{array}$ \\
\hline
\end{tabular}

\footnotetext{
SST: saúde e segurança no trabalho.

b $P<0,001$.

c $P<0,01$.

d $P<0,05$.
} 
empresa $(\beta=0,70 ; P<0,001)$ e dos trabalhadores $(\beta=0,85 ; P<0,01)$ se associaram independentemente com o grau de implantação do PSST. A tabela mostra ainda que este resultado não se modificou com a introdução das variáveis de contexto, como o nível de escolaridade da pessoa focal e dos trabalhadores, os salários dos trabalhadores, o grupo de risco da empresa e o porte. Os modelos, embora estatisticamente significativos (tabela 4), não foram, em geral, fortemente explicativos do grau de implantação, com $R^{2}$ variando entre 0,3675 , para o modelo ajustado por todas as covariáveis, a 0,4102 , para o modelo apenas com os fatores impulsionadores globais.

\section{DISCUSSÃO}

Os resultados deste estudo revelaram que, em 1 ano do ciclo de intervenção do PSST proposto e executado pelo SESI, a maioria das empresas alcançou grau de implantação intermediário, enquanto duas $(2,6 \%)$ não tiveram operacionalização relevante. A constatação de que nem todas as empresas alcançaram a implantação completa do PSST não foi uma surpresa, considerando-se as dificuldades conhecidas de desenvolvimento de ações de saúde e segurança no âmbito de empresas, apesar, neste caso, da iniciativa de contratação da prestadora para essa atividade. Essa aparente contradição pode ser compreendida quando se verifica que as decisões relativas à contratação de serviços de saúde e segurança ocupacional são reativas, comumente feitas após a ocorrência de inspeções ou do recebimento de multas ou outras penalidades $(15,19,20)$. Por outro lado, o PSST desenvolvido pelo SESI Bahia foi idealizado a partir de um padrão que envolvia profissionais qualificados e um modelo organizado na perspectiva da saúde coletiva, contrapondo-se a iniciativas cartoriais, de baixo custo, oferecidas por empresas privadas que disponibilizam serviços que enfocam o PPRA e o PCMSO.

A incompleta implantação do PSST depois de 1 ano de atividades programadas é consistente com os achados de Miranda e Dias (10) que, ao analisarem dados de inspeções de empresas conduzidas pela Delegacia Regional do Trabalho do Ministério do Trabalho na Bahia, encontraram baixa qualidade técnica dos programas de SST existentes. Essa baixa qualidade técnica ficava evidente nas inconsistências entre os pro- cedimentos previstos pelas normas em até $57 \%$ das empresas analisadas, sendo que apenas $10 \%$ cumpriam as recomendações. Além disso, foi bastante precária a participação dos trabalhadores nas ações de saúde e segurança, inclusive o envolvimento da Comissão Interna de Prevenção de Acidentes (CIPA).

Depreende-se dos resultados deste estudo que os fatores das diversas dimensões analisadas, isto é, empresa, trabalhadores e equipe de SST foram, separadamente, impulsionadores da implantação. Não houve evidências de interações estatísticas entre essas dimensões. Isso quer dizer que cada dimensão influenciou positivamente o grau de implantação, e que, para isso, uma não dependeu necessariamente da outra. Este resultado contraria a ideia de que a implantação depende da atuação sinérgica e interativa desses fatores. Entretanto, o pequeno número amostral é limitado para inferências conclusivas, valendo apenas como uma indicação que poderá ser verificada em estudos mais apropriados a análises de subgrupos.

Como mencionado anteriormente, são raros os estudos sobre determinantes da implantação de programas de SST. Em um dos poucos estudos disponíveis, 1789 empresas foram analisadas para verificar o nível de implementação de uma política de SST na Noruega em um período de 10 anos (15). Os autores constataram que $25 \%$ das firmas não se encontravam em conformidade com o marco regulatório em um item ou mais, estando em estágio avançado apenas $4 \%$. Os principais fatores identificados foram treinamento dos gestores e representantes dos trabalhadores, a realização de avaliações e atividades de planejamento.

A importância da autonomia da gestão orçamentária para a implantação dos programas de SST encontrada neste estudo não foi relatada ou analisada em outras investigações. Como isso depende em grande medida dos modelos de gestão das próprias empresas, é possível que isso reflita especificidades das normas de saúde e segurança brasileiras. Por exemplo, as recomendações mais recentes para a gestão da saúde e segurança indicam a incorporação dessas ações às do próprio negócio, o que se sustenta na ideia de que ambientes de trabalho saudáveis e seguros contribuem para a produtividade, tanto pela redução de faltas no trabalho, quanto pela maior satisfação dos trabalhadores $(1,21)$. A separação das ações de
SST em um programa dissociado da gestão geral da empresa pode resultar, ou mesmo expressar em si mesma, um papel secundário desse tipo de atuação, assim como uma falta de comprometimento da empresa. Ademais, as normas existentes contribuem para essa separação, ao recomendar programas separados e independentes para a segurança e para a saúde. Vale notar que o financiamento, a autonomia da gestão desses recursos e a liberação do trabalhador para a participação foram fundamentais para o sucesso na implantação do programa. Esses aspectos, portanto, devem fazer parte das negociações iniciais prévias à implantação.

Um outro fator importante para a implantação do PSST foi o conhecimento e o treinamento do gestor e dos trabalhadores em relação a SST. Esses fatores são classicamente considerados cruciais para qualquer tipo de estratégia de intervenção, mesmo fora do âmbito da saúde (22). Embora pareça óbvio que as pessoas se engajarão mais efetivamente em ações quando conhecem os objetivos, significados e alcance dessas ações, e se de fato contribuíram para a sua concepção e execução, isso nem sempre é a realidade dos modelos lógicos das intervenções em saúde. Comumente, os processos de planejamento e programação são de natureza normativa, ou prescritiva, com normas e procedimentos recomendados para aplicação pelas empresas de forma acrítica. Estudos sugerem que o conhecimento, por parte dos empresários e trabalhadores, sobre a segurança e a saúde e sobre o potencial de prevenção dos agravos em locais de trabalho é ainda muito pequeno, mesmo em empresas de grande porte ou de maior risco ocupacional (23). Este último estudo (23), que analisou a percepção de profissionais de saúde ocupacional sobre os fatores que influenciam a não-implantação de ações de SST em pequenas e médias empresas, identificou dificuldades de acesso a capital e crédito, falta de conhecimento dos empresários sobre a legislação e as recomendações técnicas e crença de que tais intervenções desviam o foco dos negócios. Além disso, foram também citadas a precariedade da infraestrutura física, de equipamentos e pessoal, e a não associação das empresas em órgãos de classe. Vários estudos têm demonstrado que o grau de implantação de programas desse tipo é menor nas empresas de pequeno porte (15), como as 
que predominaram neste estudo. Investigações têm apontado a necessidade de desenvolver estratégias de intervenção distintas para esse grupo de empresas (24), que, em contexto de vulnerabilidade econômica, se defrontam com o dilema entre a sobrevivência do negócio e a aplicação estrita da lei em função dos recursos escassos (25).

A integração das equipes de saúde e de segurança traz à tona um importante foco de tensão, tradicional no campo da saúde do trabalhador, nutrido pela separação dos campos disciplinares da higiene e segurança, por um lado, e da medicina do trabalho, por outro, reforçado pelos instrumentos legais. Isso vem se sustentando apesar do avanço das discussões em torno do tema, tanto na academia como nos serviços, e apesar da incorporação de profissionais com formações e experiências distintas, a exemplo da odontologia, psicologia, fonoaudiologia e serviço social, dentre outras. Contudo, o diálogo e a construção de uma intervenção interdisciplinar, ponto focal da saúde do trabalhador, ainda está por se concretizar satisfatoriamente.

Cabe destacar que, segundo o modelo lógico da intervenção, o diagnóstico epidemiológico elaborado pela prestadora é ferramenta importante, mas se observa que não tem sido utilizado como base para a ampliação de práticas de educação coletivas, componente importante para execução do PCMSO, já que as ações preventivas coletivas são ainda escassas e ainda mostram uma prática individualizada e medicalizada. As associações de empresas por ramo de atividade podem reforçar o compromisso com a sua segurança, definindo-se riscos comuns a serem enfrentados, utilizando estratégias de grupo para a socialização e a divulgação das informações $(5,11)$. Tal estratégia po- deria possibilitar maior divulgação do programa junto aos trabalhadores. Ainda que essa tarefa caiba prioritariamente à empresa, a indução e o estímulo são também responsabilidade da prestadora.

Os resultados deste estudo devem ser vistos com precaução devido a alguns limites metodológicos. Os dados provêm de rotinas administrativas e não foram coletados especificamente com a finalidade de pesquisa. Foi grande o número de informantes envolvidos, o que, por um lado, poderia provocar diferenças de qualidade ou de pontos de vista dos observadores; porém, também traz vantagens, considerando que o envolvimento de diferentes atores permite a composição de um quadro mais abrangente da realidade das empresas. Embora o número de empresas tenha sido pequeno, o uso de variáveis contínuas e a regressão linear múltipla permitiram um adequado poder estatístico ao estudo, como pode ser visto nas diversas associações estatisticamente significativas.

Em um contexto de incipiente implementação de programas de saúde e segurança no trabalho, de pouca informação sobre a cobertura dos trabalhadores por esses programas e de pequena participação das empresas, torna-se necessária a geração de conhecimento sobre a extensão e a qualidade das intervenções em saúde do trabalhador no país (26). Esse conhecimento não deve se restringir apenas aos fatores impulsionadores, mas deve focalizar também o conteúdo dos programas, processos, resultados e impactos alcançados na perspectiva do aperfeiçoamento das políticas e programas nesse campo.

\section{CONCLUSÕES}

As conclusões deste estudo devem ser consideradas com precaução a partir dos seus limites metodológicos. Embora esta pesquisa tenha avançado o conhecimento sobre os determinantes da implantação de um programa de saúde e segurança no trabalho, possivelmente um dos poucos existentes no país, o número de empresas analisadas foi pequeno, reduzindo o poder estatístico do estudo. Além disso, a natureza dos dados de rotina dos serviços resultou em simplicidade com vistas à garantia da viabilidade operacional, reduzindo, portanto, o escopo das informações disponíveis para análise.

Os estudos avaliativos têm um caráter estratégico para o campo da saúde do trabalhador, ao possibilitarem diretrizes que garantam maior equidade e justiça no acesso aos serviços de proteção à saúde. A implantação da Rede Nacional de Saúde do Trabalhador (RENAST), que compreende Centros de Referência em Saúde do Trabalhador (CERESTs) e várias ações de vigilância à saúde do trabalhador, algumas delas articuladas à atenção básica de saúde, sob a responsabilidade do Sistema Único de Saúde (SUS), poderá ter um papel importante nesta tarefa. Assim, políticas e programas de saúde, profissionais, empregadores e trabalhadores poderão agir coordenadamente, modificando o perfil de carência e a insuficiência das ações de proteção à saúde dos trabalhadores, que continuam sofrendo no exercício dos seus direitos.

Agradecimentos. Este estudo foi financiado pelo Serviço Social da Indústria (SESI), Departamento Regional da Bahia, Salvador (BA), Brasil.

\section{REFERÊNCIAS}

1. Alli BO. Fundamental principles of occupational health and safety. Genebra: International Labor Organization; 2001.

2. Brasil, Ministério do Trabalho e Emprego. NR 9 - Programa de prevenção de riscos ambientais. Portaria SSST $n^{\circ} 25,29$ de dezembro de 1994. Disponível em: www.mte.gov.br/ legislacao/normas_regulamentaDORAS/ $\mathrm{nr}_{-}$ 09_at.pdf. Acessado em janeiro de 2009.

3. Brasil, Ministério do Trabalho e Emprego. NR 7 - Programa de controle médico de saúde ocupacional. Portaria SSST $\mathrm{n}^{\circ} 24$, de 29 de dezembro de 1994. Disponível em: www. mte.gov.br/legislacao/normas_regulamenta
DORAS/nr_07_at.pdf. Acessado em janeiro de 2009.

4. DeJoy DM, Bryan SS, Wilson MG, Vandenberg RJ, Butts MM. Creating safer workplaces: assessing the determinants and role of safety climate. J Safety Res. 2004;35(1):81-90.

5. Ito A, Sakai K, Kogi K. Development of interactive workplace improvement programs in collaboration with trade associations of smallscale industries. Ind Health. 2006;44(1):83-6.

6. Kogi K. Advances in participatory occupational health aimed at good practices in small enterprises and the informal sector. Ind Health. 2006;44(1):31-4.
7. Spangenberg S, Baarts C, Dyreborg J, Jensen L, Kines P, Mikkelsen KL. Factors contributing to the differences in work related injury rates between Danish and Swedish construction workers. Safety Sci. 2003;41(6):517-30.

8. Conway H, Simmons J, Talbert T. Effects of occupational medical surveillance programs as perceived by respondents to the occupational safety and health administration's 1990-1991 Survey. J Occup Med. 1993;35(7): 687-97.

9. Pelletier KR. A review and analysis of the clinical and cost-effectiveness studies of comprehensive health promotion and disease 
management programs at the worksite: update VI 2000-2004. J Occup Environ Med. 2005;47(10):1051-8.

10. Miranda CR, Dias CR. PPRA/PCMSO: auditoria, inspeção do trabalho e controle social. Cad Saude Publica. 2004;20(1):224-32.

11. Gonçalves CGO, Iguti AM. Análise de programas de preservação da audição em quatro indústrias metalúrgicas de Piracicaba, São Paulo, Brasil. Cad Saude Publica. 2006;22(3): 609-18.

12. Santana VS, Carvalho LC, Santos CP, Andrade C, D'Oca G Morbidade em candidatos a emprego na região metropolitana de Salvador, Bahia, Brasil. Cad Saude Publica. 2001; 17(1):107-15.

13. Donabedian A. Criteria and standards for quality assessment and monitoring. QRB Qual Rev Bull. 1986;12(3):99-108.

14. Denis J, Champagne F. Análise da implantação de programas. Em: Hartz ZMA, org. Avaliação em saúde: dos modelos conceituais à prática na análise da implantação de programas. Rio de Janeiro: Editora Fiocruz; 1997. Pp. 49-88.

15. Saskvik PO, Torvatn H, Nytro K. Systematic occupational health and safety work in Norway: a decade of implementation. Safety Sci. 2003;41(9):721-38.

16. Rossi PH, Lipsey MW, Freeman HE. Evaluation: a systematic approach. Beverly Hills: Sage; 2004.
17. Fundação Kellogg. Logic model development guide. Disponível em: http://www.wkkf. org/Pubs/Tools/Evaluation/Pub3669.pdf. Acessado em fevereiro de 2006.

18. Bolfarine $H$, Bussab WO. Elementos de amostragem. São Paulo: Ed. Edgard Blucher; 2005.

19. Baumecker IC, Faria MP. Private and state interventions in safety and health at work. Em: Dwyer $\mathrm{T}$, ed. Occupational safety and health in Brazil. OSH \& Developments. Estocolmo: Swedish Association for Occupational and Environmental Health \& Development (UFA)/ Scientific Committee on "Occupational Health and Development" of the International Commission of Occupational Health (ICOH)/ Swedish National Institute for Working Life (NIWL); 2006. Pp. 9-22. (No. 8, dezembro de 2006). Disponível em: www.ufa.se/ publikationer/oshd8.pdf. Acessado em janeiro de 2009.

20. Vasconcellos LCF, Ribeiro FS. Investigação epidemiológica e intervenção sanitária em saúde do trabalhador: o planejamento segundo bases operacionais. Cad Saude Publica. 1997;13(2): 269-75.

21. World Health Organization. Guidelines for implementing healthy workplaces. Regional Office for the Western Pacific. Genebra: WHO; 1999.

22. Colligan MJ, Cohen A. The role of training in promoting workplace safety and health. Em:
Barling J, Frone MR, eds. The psychology of workplace safety. Washington D.C.: American Psychological Association; 2004. Pp. 223-248

23. Andrade LRB. Estratégias para desenvolvimento de ações de saúde e segurança no trabalho em pequenas e médias empresas. Em: Salim CA, Carvalho LF, Freitas MMC, Freitas $\mathrm{M}$, org. Saúde e segurança no trabalho, novos olhares e saberes. Belo Horizonte: Fundacentro/Universidade Federal de São João del-Rei; 2003. Pp. 251-9.

24. Takeyama H, Itani T, Tachi N, Takanishi T, Inoue $\mathrm{T}$, Murata $\mathrm{K}$, et al. A case study on evaluations of improvements implemented by WISE projects in the Philippines. Indust Health. 2006;44(1):53-57.

25. Carpentier-Roy MC, Simard M, Marchand A, Ouellet F. Towards a new model of intervention for occupational health in small firms. Ind Rel. 2001;56(1):165-94.

26. Santana VS. Workers' health in Brazil: graduate research. Rev Saude Publica. 2006;40(n esp): 101-11.

Manuscrito recebido em 27 setembro de 2007. Aceito em versão revisada em 16 de abril de 2008 .
ABSTRACT

Determinants in an occupational health and safety program implementation

Key words
Objective. To identify predictors for the degree to which a program that integrates occupational health surveillance with labor safety, and involves occupational health/ safety specialists, company management, and employees, is implemented.

Method. This ecological study evaluated companies implementing the occupational health and safety program (OHSP) proposed by the state of Bahia's regional department of Serviço Social da Indústria (Social Services for Industry, SESI) during the 2005-2006 cycle. The companies that participated were randomly selected. Data were collected through interviews with key contacts within the companies and from technical reports issued by SESI. Multiple linear regression was used to identify factors related to the company, employee, occupational/safety specialist, and any subdimensions that might promote OHSP implementation.

Results. Of the 78 companies selected (3 384 employees), the degree to which OHSP was implemented was "advanced" in $24.4 \%$, "intermediate" in $53.8 \%$, and "initial" in $19.3 \%$. Company-related, employee-related and specialist-related factors were positively associated with OHSP implementation $(P<0.001)$. The most important factor overall was the program's financial autonomy $(\beta=4.40 ; P<0.001)$. Bivariate analysis revealed that the degree of implementation was associated with the employees' level of health/safety knowledge $(\beta=1.58 ; P<0.05)$ and training $(\beta=0.40 ; P<0.001)$ and with communication between the occupational safety team $(\beta=1.89 ; P<0.01)$ and the health team $(\beta=0.58 ; P<0.05)$. These findings remained unchanged after adjustment for levels of education among managers and employees, salary/wages, company size, and risk.

Conclusions. The time and resources available for employees to dedicate to occupational health and safety, the integration and reinforcement of employee and manager training programs, and improved relationship between occupational health and safety teams may contribute to the success of health and safety promotion efforts among employees.

Occupational health, safety, safety management, surveillance, Brazil. 